\title{
Adaptor proteins of type VI secretion system effectors
}

6

7

8

9

Daniel Unterweger ${ }^{1 \#}$, Benjamin Kostiuk $^{1}$ \& Stefan Pukatzki ${ }^{1,2} *$

10

11

$12{ }^{1}$ Department of Medical Microbiology and Immunology, University of Alberta,

13 Edmonton, AB T6G 2S2, Canada

$14 \quad{ }^{2}$ Department of Immunology \& Microbiology, University of Colorado School of Medicine,

15 Aurora, CO 80045, USA

$16{ }^{\#}$ Current address: Department of Zoology, University of Oxford, Oxford OX1 3PS, United

17 Kingdom

18

19

20 *Corresponding author: Pukatzki, S. (stefan.pukatzki@ucdenver.edu)

21

22 Keywords (2-6): Type VI secretion system, adaptor protein, accessory protein, chaperone,

23 effector translocation, diversity 
24 Abstract (max 50 words, currently 46)

25 Bacteria use the type VI secretion system (T6SS) to kill neighboring cells. One key feature of the

26 T6SS is the secretion of diverse effectors. Here, we discuss six publications that describe three

27 superfamilies of T6SS proteins, each dedicated to mediate the secretion of cognate effectors.

28

29

30

31

32

33

34

35

36

37

38

39

40

41

42

43

44

45 


\section{Text}

47 The type VI secretion system (T6SS) is a contractile puncturing device, enabling bacteria to kill

48 neighboring prokaryotic and eukaryotic cells in a contact-dependent manner. Contraction of a

49 cytoplasmic outer sheath results in the ejection of an inner tube (comprised of Hcp subunits) that

50 can penetrate into a neighboring cell [1]. Effectors are found within the tube and on top of the tip

51 of the tube. Once translocated, the effectors act in a toxic manner unless inhibited by T6SS

52 immunity proteins produced by the receiving cell. T6SS effectors differ in the mechanism by

53 which they associate with the tip of the T6SS machinery tube, as well as in their biochemical

54 activity. The first T6SS effectors to be described were the so-called valine glycine-rich proteins

$55 \mathrm{G}(\mathrm{VgrG})$ that exhibit structural homology to the cell-puncturing device of T4 bacteriophage,

56 establishing the tip of the T6SS. VgrG proteins consist of a core that can be fused to a C-terminal

57 domain with effector activity [2]. In addition, VgrGs contain a region that binds the so-called

58 PAAR-domain (proline-alanine-alanine-arginine domain) of a defined class of effector proteins

59 [1]. To define the mechanisms by which diverse T6SS effectors are translocated, we summarize

60 the recent characterization of proteins with domains of unknown function (DUFs) that have been

61 labeled as accessory [3], adaptor [4], or chaperone proteins [5; 6].

62 These adaptors are responsible for the secretion of effectors with or without PAAR domains.

63 Liang et al. and Unterweger et al. independently described a member of the superfamily of

64 proteins with a conserved DUF4123 domain required for the secretion of effectors lacking a

65 PAAR domain in Vibrio cholerae [4; 6]. Diniz et al., Whitney et al. and Cianfanelli et al.

66 characterized representatives of the superfamily of proteins with a conserved DUF1795 domain

67 that are needed for the secretion of PAAR-domain effectors in Serratia marcescens and

68 Pseudomonas aeruginosa [3; 5; 7]. More recently, Bondage et al. identified a third group of 
69 adaptor proteins characterized by the DUF2169 in Agrobacterium tumefaciens [8]. Adaptor

70 proteins representing three superfamilies that have been studied experimentally are compiled in

71 Table 1 along with the effectors they translocate and other pertinent information.

72

73 Table 1. Experimentally studied adaptor proteins subdivided by DUF superfamily

74

Superfamily Adaptor Effector $\begin{gathered}\text { Effector } \\ \text { activity }\end{gathered} \begin{gathered}\text { Effector- } \\ \text { region for } \\ \text { VgrG } \\ \text { interaction }\end{gathered} \quad$ Species Reference

\begin{tabular}{|c|c|c|c|c|c|c|}
\hline \multirow[t]{3}{*}{ DUF1795 } & EagR1 & Rhs1 & Unknown & $\begin{array}{l}\text { PAAR- } \\
\text { domain }\end{array}$ & S. marcescens & 3 \\
\hline & EagR2 & Rhs2 & DNAse & $\begin{array}{l}\text { PAAR- } \\
\text { domain }\end{array}$ & S. marcescens & 7 \\
\hline & EagT6 & Tse6 & $\begin{array}{c}\operatorname{NAD}(P)^{+} \\
\text {Glycohydrolase }\end{array}$ & $\begin{array}{l}\text { PAAR- } \\
\text { domain }\end{array}$ & P. aeruginosa & 5 \\
\hline DUF2169 & Atu3641 & Tde2 & DNAse & unknown & A. tumefaciens & 8 \\
\hline \multirow[t]{4}{*}{ DUF4123 } & $\begin{array}{l}\text { Tap-1 } \\
\text { (TecL) }\end{array}$ & TseL & Lipase & unknown & V. cholerae & 4,6 \\
\hline & VasW & VasX & Pore formation & unknown & V. cholerae & 10 \\
\hline & TecC & TseC & Unknown & unknown & A. hydrophila & 6 \\
\hline & $\begin{array}{c}\text { Tap-1 } \\
\text { (Atu4349) }\end{array}$ & Tde1 & DNAse & unknown & A. tumefaciens & 8 \\
\hline
\end{tabular}

75

76

77 Biochemical studies of the proteins belonging to the DUF4123 and DUF1795 superfamilies have

78 demonstrated direct interactions with their cognate effectors are required form protein complexes

79 with VgrG proteins [3-6; 8]. The observation that some PAAR domain-containing effectors are

80 unable to bind to VgrG by themselves further supports the notion that DUF1789 proteins are

81 required for the stability and three-dimensional conformation of effectors. Indeed, adaptor 
82 proteins of all three superfamilies were reported to stabilize their cognate effector indicating that

83 these proteins act as chaperones [5;8;9]. Genetic and biochemical experiments indicate that

84 DUF4123, DUF1795 and DUF2169 proteins are also required for the secretion of cognate

85 effectors and subsequent killing of targeted prey bacteria [3-8]. Importantly, DUF4123,

86 DUF1795 and DUF2169 proteins are only required for the delivery of their cognate effector, but

87 not for the functionality of the T6SS $[3-6 ; 8 ; 10]$. Thus, adaptor proteins of the three

88 superfamilies differ in this aspect from Hcp proteins, which form the inner T6SS tube and can

89 act as chaperones for small effectors therein [11].

90 Experiments conducted by Whitney et al. suggest that DUF1795 protein EagT6 of $P$. aeruginosa

91 binds to the N-terminal half of PAAR domain-containing effector Tse6 - thereby stabilizing Tse6

92 [5]. The DUF1795 superfamily protein EagR1 has been described to interact also with the N-

93 terminal domain of its cognate effector, Rhs1, in S. marcescens [7]. The crystal structure of

94 EagT6 resembles the shape of a horseshoe and the authors proposed a model in which the

95 chaperone EagT6 shields hydrophobic transmembrane helices of Tse6 during the translocation

96 from one bacterium to another [5]. However, the DUF1795 superfamily proteins of $S$.

97 marcescens do not appear to accompagny their secreted effectors as they are not detected in

98 supernatants analyzed by mass spectrometry [7]. Western-blot analysis of culture supernatants

99 also failed to detect the DUF4123 protein Tap-1 (TecL) or DUF2169 protein Atu3641 in culture

100 supernatants $[4 ; 6 ; 8]$. As a result, DUF4123 proteins were proposed to load their cognate

101 effector onto the secretion system without being secreted themselves (see Figure 1). With the

102 adaptor gene deleted, the effector is no longer detected at the tip of the T6SS and vice versa,

103 indicating that the two proteins interact prior to loading onto the tip $[4 ; 7 ; 8]$. Effectors loaded by

104 the DUF4123 superfamily proteins interact with sites of VgrG proteins situated C-terminal to the 
105 highly conserved core and distinct from the VgrG-site interacting with PAAR domain effectors

$106[4 ; 8]$. Determining the structure and function of the various conserved DUF domains is needed

107 to better understand the differences between the superfamilies of adaptor proteins.

108 Strains of the same bacterial species can contain a repertoire of multiple effectors that differ in

109 their biochemical activity and the mechanism by which they are associated with the T6SS.

110 Bacteria such as $S$. marcescens produce various effectors that compete for a binding site at the

111 T6SS tip to be transported in the same translocation event [7]. Differences in the affinity of the

112 adaptor proteins to the T6SS tip might enable an adaptor protein with high affinity to load its

113 cognate effector onto the T6SS at higher frequency [7]. Another way by which the adaptor

114 protein might influence the effector set of a particular bacterium is if the adaptor protein-

115 encoding gene aids in the acquisition of a new effector protein-encoding gene. The effector

116 repertoire of a bacterial strain may change due to the acquisition of effector protein-encoding

117 genes in effector modules that are likely exchanged between strains via horizontal gene transfer

118 [12]. These effector modules contain all or part of an adaptor protein-encoding gene, an effector

119 protein-encoding gene, and an immunity protein-encoding gene [4]. The genetic linkage of genes

120 encoding DUF4123, DUF1795 and DUF2169 superfamily proteins with genes encoding their

121 cognate effectors located immediately downstream suggests that this genetic organization applies

122 to all three superfamilies $[3 ; 4 ; 6 ; 8]$. Homologous recombination at conserved sites in the center

123 of tap- 1 and an additional site flanking the 3 '-end of the module would result in the formation of

124 chimeric adaptor proteins that are specific to their cognate effector and match the resident VgrG-

1251 protein encoded upstream of the adaptor gene [4]. Adaptor proteins might thereby play a

126 crucial role for providing a system in which newly acquired effectors, which are encoded on 
127 effector modules together with at least parts of the adaptor protein-encoding gene, are 128 successfully translocated by the resident T6SS.

129 The ability to secrete a variety of effectors makes the T6SS a versatile system, aiding bacteria in 130 competition with prokaryotic and eukaryotic competitors. Effector diversity is the basis for the 131 formation of compatibility groups: bacteria with the same set of effectors and immunity genes 132 are able to co-exist in direct contact; incompatible strains with differing effectors kill each other 133 due to the lack of cognate immunity proteins [12]. Multiple mechanisms to associate effectors 134 with the tip of the T6SS may increase the number of effectors and the variety of effector proteins 135 with differing toxic activities that can be translocated during the same translocation event. 136 DUF4123, DUF1795 and DUF2169 superfamily proteins are widespread among bacterial 137 species [3-6;8] and are thus likely to make crucial contributions to the competitive fitness of 138 bacteria. Studying the basic mechanism of effector secretion has implications for our basic 139 understanding of microbial competition and could lead to the development of novel therapeutic 140 interventions that instruct pathogens to outcompete each other. 


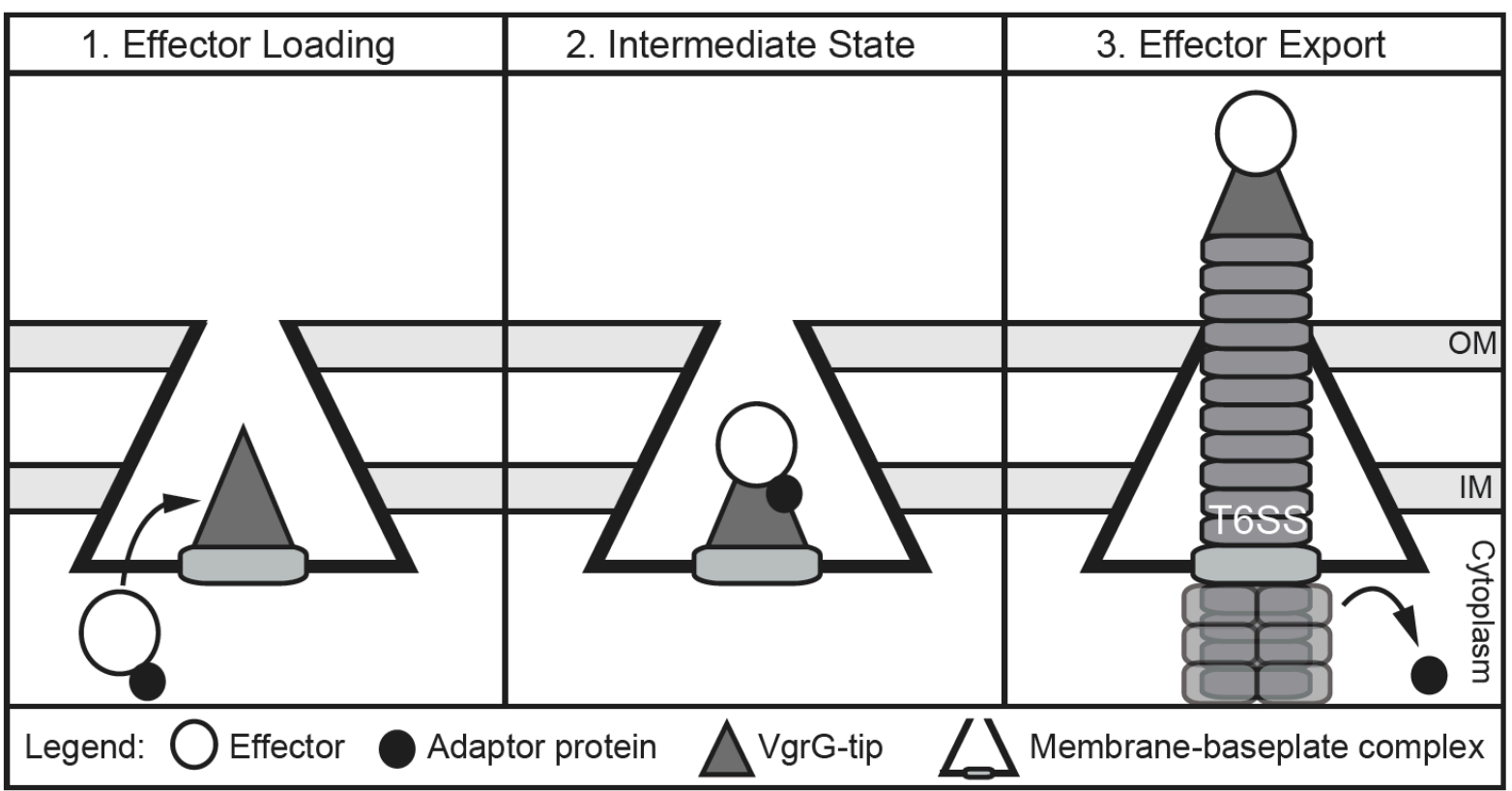

144 Figure 1. General model for the role of adaptor proteins in T6SS-mediated export.

145 The membrane-baseplate complex and the Hcp tube secreted upon contraction of the VipA and

146 VipB sheath are depicted. Adaptor proteins with characteristic DUF domains stabilize cognate

147 effector proteins and are required for effector proteins to locate to the tip of the T6SS. The

148 current working model suggests that once located to the tip, the effector is getting exported via

149 the T6SS whereas the adaptor protein stays behind. IM inner membrane, OM outer membrane. 


\section{References}

152 [1] Cianfanelli, F.R., Monlezun, L., and Coulthurst, S.J. (2016). Aim, Load, Fire: The Type VI 153 Secretion System, a Bacterial Nanoweapon. Trends in microbiology 24, 51-62.

154 [2] Pukatzki, S., Ma, A.T., Revel, A.T., Sturtevant, D., and Mekalanos, J.J. (2007). Type VI secretion system translocates a phage tail spike-like protein into target cells where it cross-links actin. Proceedings of the National Academy of Sciences of the United States of America 104, $157 \quad 15508-15513$.

158 [3] Alcoforado Diniz, J., and Coulthurst, S.J. (2015). Intraspecies Competition in Serratia marcescens Is Mediated by Type VI-Secreted Rhs Effectors and a Conserved EffectorAssociated Accessory Protein. Journal of bacteriology 197, 2350-2360.

161 [4] Unterweger, D., Kostiuk, B., Otjengerdes, R., Wilton, A., Diaz-Satizabal, L., and Pukatzki, 162 S. (2015). Chimeric adaptor proteins translocate diverse type VI secretion system effectors in 163 Vibrio cholerae. The EMBO journal 34, 2198-2210.

164 [5] Whitney, J.C., Quentin, D., Sawai, S., LeRoux, M., Harding, B.N., Ledvina, H.E., Tran, 165 B.Q., Robinson, H., Goo, Y.A., Goodlett, D.R., et al. (2015). An interbacterial NAD(P)(+) glycohydrolase toxin requires elongation factor $\mathrm{Tu}$ for delivery to target cells. Cell 163, 607-619. [6] Liang, X., Moore, R., Wilton, M., Wong, M.J., Lam, L., and Dong, T.G. (2015). Identification of divergent type VI secretion effectors using a conserved chaperone domain. Proceedings of the National Academy of Sciences of the United States of America 112, 91069111.

[7] Cianfanelli, F.R., Alcoforado Diniz, J., Guo, M., De Cesare, V., Trost, M., and Coulthurst, S.J. (2016). VgrG and PAAR Proteins Define Distinct Versions of a Functional Type VI Secretion System. PLoS pathogens 12, e1005735.

[8] Bondage, D.D., Lin, J.S., Ma, L.S., Kuo, C.H., and Lai, E.M. (2016). VgrG C terminus confers the type VI effector transport specificity and is required for binding with PAAR and adaptor-effector complex. Proceedings of the National Academy of Sciences of the United States of America 113, E3931-3940.

178 [9] Ma, L.S., Hachani, A., Lin, J.S., Filloux, A., and Lai, E.M. (2014). Agrobacterium tumefaciens deploys a superfamily of type VI secretion DNase effectors as weapons for interbacterial competition in planta. Cell host \& microbe 16,94-104. [10] Miyata, S.T., Unterweger, D., Rudko, S.P., and Pukatzki, S. (2013). Dual expression profile of type VI secretion system immunity genes protects pandemic Vibrio cholerae. PLoS pathogens 9, e1003752.

184 [11] Silverman, J.M., Agnello, D.M., Zheng, H., Andrews, B.T., Li, M., Catalano, C.E., Gonen, 185 T., and Mougous, J.D. (2013). Haemolysin coregulated protein is an exported receptor and chaperone of type VI secretion substrates. Molecular cell 51, 584-593. diverse effector modules for intraspecific competition. Nature communications 5, 3549. 